\title{
Risk aversion, intergenerational equity and climate change
}

\author{
Minh Ha-Duong* $\quad$ Nicolas Treich ${ }^{\dagger}$
}

July 11, 2003

\begin{abstract}
The paper investigates a climate-economy model with an iso-elastic welfare function in which one parameter $\gamma$ measures relative risk-aversion and a distinct parameter $\rho$ measures resistance to intertemporal substitution. We show both theoretically and numerically that climate policy responds differently to variations in the two parameters. In particular, we show that higher $\gamma$ but lower $\rho$ leads to increase emissions control. We also argue that climate-economy models based on intertemporal expected utility maximization, i.e. models where $\gamma=\rho$, may misinterpret the sensitivity of the climate policy to risk-aversion.
\end{abstract}

Keywords: risk-aversion, equity, discounting, climate change

JEL classification: Q20, D81, D90, H43

\section{Introduction}

In this paper, we explore the issues of risk and intergenerational equity within a climate-economy model. Our approach differs from the recent approach that has examined what adjustments to the discount factor are necessary to account for a future environmental risk (see, e.g., [14], [4]). Instead, we examine how social preferences specification with respect to risk and equity affects the optimal climate policy. More precisely, we use a broad class of preferences that is particularly appropriate to examine risk-aversion and intertemporal substitution effects: recursive preferences. These preferences were introduced by [9]. To date, recursive preferences have been used mostly in finance and precautionary savings literature ([13], [3]).

Most previously published models of climate risk policy use the intertemporal expected utility (EU) framework. It is well-known that this framework imposes a specific restriction. With EU preferences, resistance to intertemporal substitution is constrained to be equal to relative risk-aversion. Let us recall

*CNRS-CIRED, Paris. haduong@centre-cired.fr

${ }^{\dagger}$ LEERNA-INRA, University of Toulouse 
that resistance to substitution controls the attitude toward variations in consumption across time, under certainty. On the other hand, risk-aversion controls the attitude toward variations in consumption across states of the world, at a given date. Hence the restriction is suspicious since it links conceptually two different aspects of preferences, risk-aversion and intergenerational equity. Furthermore, from a theoretical viewpoint this restriction prevents consideration of plausible situations where society would display high risk-aversion together with low resistance to substitution, or vice versa. Another consequence is that the effect of resistance to substitution and risk-aversion cannot be conveniently investigated within the EU framework: changing the curvature of the utility function makes both resistance to substitution and risk aversion move together and this does not permit an unambiguous interpretation.

In this paper, we use recursive preferences with iso-elastic functional forms in which one parameter $\gamma$ measures risk-aversion and a distinct parameter $\rho$ measures resistance to intertemporal substitution. Our first objective will be to clarify the respective role of $\gamma$ and $\rho$ within a two-period stock pollutant model. We will show that, in general, an increase in risk-aversion $\gamma$ leads to an increase in emissions control while an increase in resistance to substitution $\rho$ leads to a decrease in emissions control. We will next examine numerically the separate effects of these two parameters in a multi-period integrated model relevant for the analysis of a policy response to the risk of global warming. Our numerical simulations support the theoretical predictions about the opposite effects of $\gamma$ and $\rho$ on optimal emissions control. A side result is that there is more variation when the parameters vary separately rather than jointly $(\gamma=\rho)$, as in the expected utility framework.

\section{Risk-aversion and substitution effects in a two- period stock pollutant model}

Consider a simple two-period model. Consumption $c_{t}$ is the only source of utility in each period, $t=1,2$. Consumption is the sum of two positive terms, $c_{t}=y_{t}+e_{t}$, where $y_{t}$ is exogenous wealth, and $e_{t}$ is the endogenous control variable which represents the level of emissions of $\mathrm{CO}_{2}$ emitted in period $t$. The initial stock of pollutant $m_{0}$ is given, and afterward it accumulates following $m_{t}=r m_{t-1}+e_{t}$ where $r>0$ denotes the fraction of emissions that remains from one period to the next. Pollution damages occur in period 2 proportionally to the stock $m_{2}$ and to a stochastic parameter $1-\widetilde{x}$. Expressed in terms of stocks, consumption stream is thus:

$$
\left(c_{1}, \widetilde{c}_{2}\right)=\left(y_{1}+m_{1}-r m_{0}, y_{2}-r m_{1}+\widetilde{x} m_{2}\right)
$$

In the recursive framework, preferences over $\left(c_{1}, \widetilde{c}_{2}\right)$ may be described as follows. Stochastic future consumption $\widetilde{c}_{2}$ is converted into its certainty equivalent $\mu\left(\widetilde{c}_{2}\right)$ given by: 


$$
\begin{aligned}
\mu\left(\widetilde{c}_{2}\right) & =v^{-1}\left(\mathrm{E} v\left(\widetilde{c}_{2}\right)\right) \\
& =\left(\mathrm{E} \widetilde{c}_{2}^{1-\gamma}\right)^{\frac{1}{1-\gamma}}
\end{aligned}
$$

where $E$ denotes the expectation operator. We thus use a specific iso-elastic utility function where $\gamma>0$ is the Arrow-Pratt relative risk-aversion parameter.

To maximize social welfare in period 1 , denoted $U_{1}$, this certainty equivalent is discounted and combined with current consumption $c_{1}$ using an iso-elastic aggregator function $f$ :

$$
\begin{aligned}
U_{1} & =f\left(c_{1}, \mu\left(\widetilde{c}_{2}\right)\right) \\
& =\left(c_{1}^{1-\rho}+\beta \mu\left(\widetilde{c}_{2}\right)^{1-\rho}\right)^{\frac{1}{1-\rho}}
\end{aligned}
$$

These preferences clearly separate risk aversion - tied to the certainty equivalent function $\mu$ or $v$ - from intertemporal substitution - tied to aggregator function $f$. Parameter $\beta$ is the pure time preference discounting factor. Parameter $\rho>0$ in the aggregator function is the resistance to intertemporal substitution parameter. When $\rho=\gamma$ we retrieve the standard intertemporal expected utility framework and the social planner simply maximizes $\left(c_{1}^{1-\gamma}+\right.$ $\left.\beta \mathrm{E}\left(\widetilde{c}_{2}{ }^{1-\gamma}\right)\right)^{\frac{1}{1-\gamma}}$.

The objective is to find the optimal resource use policy $\left(m_{1}, m_{2}\right)$ which maximizes $U_{1}$, bringing (2), (3) and (1) together. Annex 1 shows that the optimal consumption level in the first period is:

$$
c_{1}^{*}(\gamma, \rho)=\frac{y_{2} / r+y_{1}-r m_{0}}{1+\beta^{1 / \rho}(\mu[\gamma] r)^{1 / \rho-1}}
$$

where $\mu[\gamma]$ is a positive parameter defined eq. 12 that will be interpreted later using eq. 5 .

Having solved analytically the model, one can easily notice that consumption in period 1 is increasing in $y_{t}, t=1,2$, and decreasing in the decay factor $r$, in the initial pollution level $m_{0}$ and in the discount factor $\beta$.

This closed-form solution allows us to show theoretically how the optimal consumption depends in a different manner on $\rho$ and $\gamma$. The total differential of $c_{1}^{*}$ with respect to $(\gamma, \rho)$ is:

$$
d c_{1}^{*}=\underbrace{\frac{\partial c_{1}^{*}}{\partial \gamma} d \gamma}_{\text {Risk-aversion effect }}+\underbrace{\frac{\partial c_{1}^{*}}{\partial \rho} d \rho}_{\text {Resistance to substitution effect }}
$$

Comparative statics in the EU framework amounts to examining the previous differential only along the $\rho=\gamma$ direction. In this way risk-aversion and substitution effects are mixed together within the EU framework. Recursive utility allows us to show in the Annex 1 that: 
- $\frac{\partial c_{1}^{*}(\gamma, \rho)}{\partial \gamma}<0$ if and only if $\rho>1$.

- $\frac{\partial c_{1}^{*}(\gamma, \rho)}{\partial \rho}>0$ if and only if $\beta r \mu[\gamma]>1$.

Let us comment briefly on these two results. First, since $\rho>1$ is in general considered as more plausible than the opposite (see more on this point later), we get that the risk-aversion effect is negative in general. This makes sense. More risk-aversion decreases current consumption and thus decreases current emissions today. This leads to reduced future exposure to a risk of climate change.

Second, direct computations show that

$$
\frac{\mu\left(\widetilde{c}_{2}^{*}\right)-c_{1}^{*}}{c_{1}^{*}}=(\beta r \mu[\gamma])^{1 / \rho}-1
$$

Hence we get that $\beta r \mu[\gamma]>1$ if and only if $\mu\left(\widetilde{c}_{2}^{*}\right)>c_{1}^{*}$. As a result, the resistance of substitution effect is positive if and only if there is positive expected growth. This last assumption is generally accepted without much discussion. The intuition behind this effect is that higher resistance to substitution raises the desire to smooth consumption over time, that is to take from the richer future generation and give to the poorer current generation. Since increasing emissions raises current consumption and thus reduces future consumption, increasing $\rho$ generally implies higher optimal first period emissions.

In short, this study shows that risk-aversion and substitution effects in general go in the opposite direction. Analyzing only the joint effect $d c_{1}^{*}(\gamma, \gamma) / d \gamma$ as in EU models theoretically underestimates the effect of risk-aversion.

\section{Climate-economy with recursive preferences}

In the previous section, with the help of a simple stock pollution model, we have analyzed the sensitivity of the optimal first-period pollution level to the resistance to intertemporal substitution $\rho$ and to relative risk aversion $\gamma$ parameters. We turn now to the empirical analysis on this question within a climate economy. The objective is two-fold. First, to examine whether the intuitions derived in the simple theoretical model carry over to a multi-period framework more representative for climate policy analysis. Second, to investigate how large are the effects generated by preferences specification with respect to risk and intergenerational equity.

The model considered below can be viewed as an extension to the DIAM model first described in [5]. The model is presented in Annex 2. It is discussed extensively in [6].

The model combines a stylized carbon-cycle with a neoclassical growth model. It is calibrated according to recent IPCC scenarios [8]. These scenarios describe future economic conditions in 2020, 2050 and 2080. As a result, we will consider four period in our model. Each period lasts for 30 years and represents a generation (e.g. generation 1 is 1990-2019). 
Production is a Cobb-Douglas function of Capital, Labor and Energy inputs. Investment is a fixed fraction of production and accumulates with a fixed depreciation rate. Labor is exogenous. Exogenous technical progress and climate change impacts directly affect the overall level of productivity in the production function. The level of Energy input depends upon its price $p_{t} X_{t}$, where $p_{t}$ is a given baseline and $X_{t}$ the endogenous energy tax multiplier. When $X_{t}$ increases, the energy used decreases. Production, consumption, and welfare decrease too, but proportionally much more slowly than energy. Less energy used translates into less $\mathrm{CO}_{2}$ emissions, hence a lower atmospheric $\mathrm{CO}_{2}$ concentration, which means ultimately less climate change impact.

This process thus represents the first order effect of carbon tax (no distortions) as it is most often represented in the integrated assessment literature. Let us make one specific comment though about equation (19). This equation stems implicitly from the assumption that the energy sector maximizes its profit blindly, in a sequence of static equilibria. The energy sector optimizes within a 30 years horizon, only the social planner has an intergenerational point of view. Given the price of output is 1 and the price of energy is $p_{t} X_{t}$, this assumption implies a well known Cobb-Douglas property

$$
Y_{t} /\left(p_{t} X_{t} E_{t}\right)=e
$$

The share of energy $e$ disappears from equation (19) because the equation at date $t$ is scaled with the equation at date 1 . Note that equation (17) is also scaled this way.

Let us now turn to the calibration of our model. There is probably no thing such as a realistic representation of climate change impact, but the version of the model used in this paper proceeds along the following lines. We assume that there are two states of the world, low damage $(L)$ with a $25 \%$ probability or high damage $(H)$ with $75 \%$ probability. For notational simplicity, we omit in the following paragraphs the index $i=H$ or $L$ that applies to all variables as the Annex 2 listing explicitly shows. The damage $F_{t}$ is expressed in terms of reduction in productivity for a doubling $\mathrm{CO}_{2}$ concentration $(550 \mathrm{ppm})$. This damage is $30 \%$ productivity loss if we are in state $H$ and only $8 \%$ in state $L$.

The baseline for results presented below is IPCC scenario SRES A2. It describes a 2080 world with 12.4 billions inhabitants (up from 5.3 in 1990), with a per capita GNP of $8.9 \mathrm{k} \$$ /year (up from $4.0 \mathrm{k} \$$ ) and an GNP energy intensity of $7.4 \mathrm{MJ} / \$$ (down from $12.8 \mathrm{MJ} / \$$ ). In this scenario, $\mathrm{CO}_{2}$ emissions in 2080 are well above $20 \mathrm{GtC}$. Importantly, this scenario is a non-mitigation scenario. The calibration has thus been made as follows. Ex ante levels of production $Y_{1}$ and $E_{1}$ are given parameters. The initial level of capital is set at three times $Y_{1}$. Control $X_{t}$ and damage $F_{t}$ were first set to unity, i.e. ignored. This allowed us to compute values for baseline energy costs $p_{t}$ and technical progress coefficients $a_{t}$ by using scenario $\mathrm{A} 2$ values for $Y_{t}$ and $E_{t}$.

All in all, the calibration of our climate economy based on a non-mitigation scenario resembles the usual methodology developed by Nordhaus for DICE [10]. Since the originality is that we introduce recursive preferences within a climate 
economy, let us now explain in more detail how we have calibrated recursive preferences.

The previous two-period model generalizes directly to a $N$-periods model as $U_{t}=f\left(c_{t}, \mu_{t}\right)$ and $\mu_{t}=v^{-1}\left(\mathrm{E}_{t} v\left(\tilde{U}_{t+1}\right)\right)$, where $\tilde{U}_{t+1}$ is next generation uncertain utility. In such a framework, each generation cares only about its own consumption and the certainty equivalent of the next generation's utility. Maximizing the welfare of the first generation $U_{1}$ insures by recursivity that the interests of all future generations are accounted for.

The choice of the relative units of consumption and utility matters in numerical models. This is dealt with by introducing a new coefficient $\alpha$ which was not apparent in the two period model (the coefficient was unity). This gives the following recursive preferences:

$$
U_{t}^{1-\rho}=\alpha c_{t}^{1-\rho}+\beta\left(\mathrm{E}_{t} \tilde{U}_{t+1}^{1-\gamma}\right)^{\frac{1-\rho}{1-\gamma}}
$$

In the literature, this coefficient is set to $\alpha=1-\beta$. In order to understand this choice of $\alpha$, let $\tilde{g}$ be the expected growth of utility beyond the last period, that is $\tilde{U}_{N+1}=(1+\tilde{g}) U_{N}$. Then equation (6) in that period is equivalent to:

$$
\alpha\left(c_{N} / U_{N}\right)^{1-\rho}=1-\beta\left[\mathrm{E}_{t}(1+\tilde{g})^{1-\gamma}\right]^{\frac{1-\rho}{1-\gamma}}
$$

It appears that the equality $\alpha=1-\beta$ arises from two stationarity conditions: $U_{4}=c_{4}$ and $\tilde{g}=0$. The first condition sets $U$ and $c$ in the same units, that is the same numerical scale. The second stationarity condition potentially leads to mathematical problems with a zero utility discount rate, as $\beta=1$ implies $\alpha=0$. However, divergence under assumptions of zero stationary growth and zero utility discount rate should not come as a surprise here, since recursive preferences generalizes classical discounted intertemporal utility.

Another important question is the inclusion of population $l_{t}$ into the social welfare criteria. In doing so, our objective was to retrieve the standard intertemporal expected utility model in the special case $\rho=\gamma$. Thus $U_{t}$ and $\mu_{t}$ are defined per capita, and the recursive equations of the model become:

$$
\begin{aligned}
U_{t}^{1-\rho} & =(1-\beta)\left(l_{t} / l_{N}\right)^{\rho} c_{t}^{1-\rho}+\beta \mu_{t}^{1-\rho} \\
\left(l_{t} \mu_{t}\right)^{1-\gamma} & =\mathrm{E}_{t}\left(l_{t+1} \tilde{U}_{t+1}\right)^{1-\gamma}
\end{aligned}
$$

The energy tax is then chosen to optimize the first's generation welfare. The risk of climate impact affects the economy from period 1 , the state of the nature $H$ or $L$ is determined at the start. However, the social planner adjusts the optimal policy only in period 4 . The reason for this lag is to account for political inertia plus the period of scientific doubt before the true level of climate impact becomes "sufficiently observable". A thorough examination of the effects of uncertainty resolution and adjustment costs is developed in [6]. Also the model assumes that the endogenous control variable $X_{t}$ is non negative. Importantly, in the final period, $X_{4}$ is chosen so as to stabilize the atmospheric $\mathrm{CO}_{2}$ concentration in period 4 for ever. 
Finally, there is the issue of the calibration of the parameters $\gamma$ and $\rho$. Research on savings and portfolio behavior analysis derived many empirical estimates. Hall [7] estimated the response of saving to the real interest rate, that is the elasticity of intertemporal substitution $(1 / \rho)$. All estimates presented by Hall report small elasticity of substitution that may well be zero. [12] argued that Hall's model was mis-specified and found estimates of resistance to substitution $\rho$ that vary between 2.2 and 3.1. Epstein and Zin's seminal paper [3] found estimates for $\rho$ that vary between 1.2 and 5 and estimates for $\gamma$ that vary between 0.8 and 1.3. Using a similar approach, [11] estimates for $\rho$ and $\gamma$ are in the range of 1.5. It is also noticeable that [11] accepted the hypothesis $\rho=\gamma$ while Epstein-Zin rejected the EU model. In an experimental framework, [2] reported estimates of each parameter based on individual responses to hypothetical situations. They found high heterogeneity among individuals. A striking result of their study is that risk aversion and resistance to substitution are not correlated at all across individuals.

To sum up, there is no decisive empirical evidence either to reject or to accept intertemporal EU models $(\rho=\gamma)$ nor to choose some specific values for $\rho$ and $\gamma$. [1] reports that 1.5 is the currently available best-guess for both parameters. Values between 0.5 and 4, with emphasis upon 1.5 and 2.0 will be used in the next section. Baseline parameters values are given in Annex 2.

\section{Risk-aversion and substitution effects in the climate economy}

This section examines the sensitivity of the optimal carbon tax to the riskaversion and the resistance to intertemporal substitution parameters.

Figure 1 displays the relative effect $\gamma$ and $\rho$ on pollution control at dates 2020, 2050 and 2080. The horizontal axis represents variations in parameters $\gamma$ and $\rho$ from 0.5 to 4 . Values corresponding to logarithmic preferences, i.e. $\gamma \rightarrow 1$ and/or $\rho \rightarrow 1$ are interpolated, we only computed the value at 1.1 and assumed continuity. The vertical scale represents the optimal energy price multiplier $X_{t}$ computed by the model described in the previous section. Hence, the optimal carbon tax is thus simply given by $X_{t}-1$.

Note also that 2080's optimal tax corresponds to two curves. This is because we allowed period 4 policy to be contingent to $H$ or $L$. More precisely, the upper (respectively lower) branch represents last period's optimal tax in state $H$ (respectively $L$ ). The intuition here is simply that the higher is the climate impact, the higher is the optimal tax rate.

Observe first that the optimal tax is increasing over time. Indeed, no control is optimal is 1990 (not displayed), then optimal control becomes a few percent or zero in 2020 while it increases up to a tax range located between $70 \%$ and $100 \%$ in 2050 . Finally, the optimal tax may go up to $250 \%$ in 2080 in state $H$ and for high values of risk-aversion.

Let us examine the effect of risk-aversion alone, that is Figure 1's left panel. 

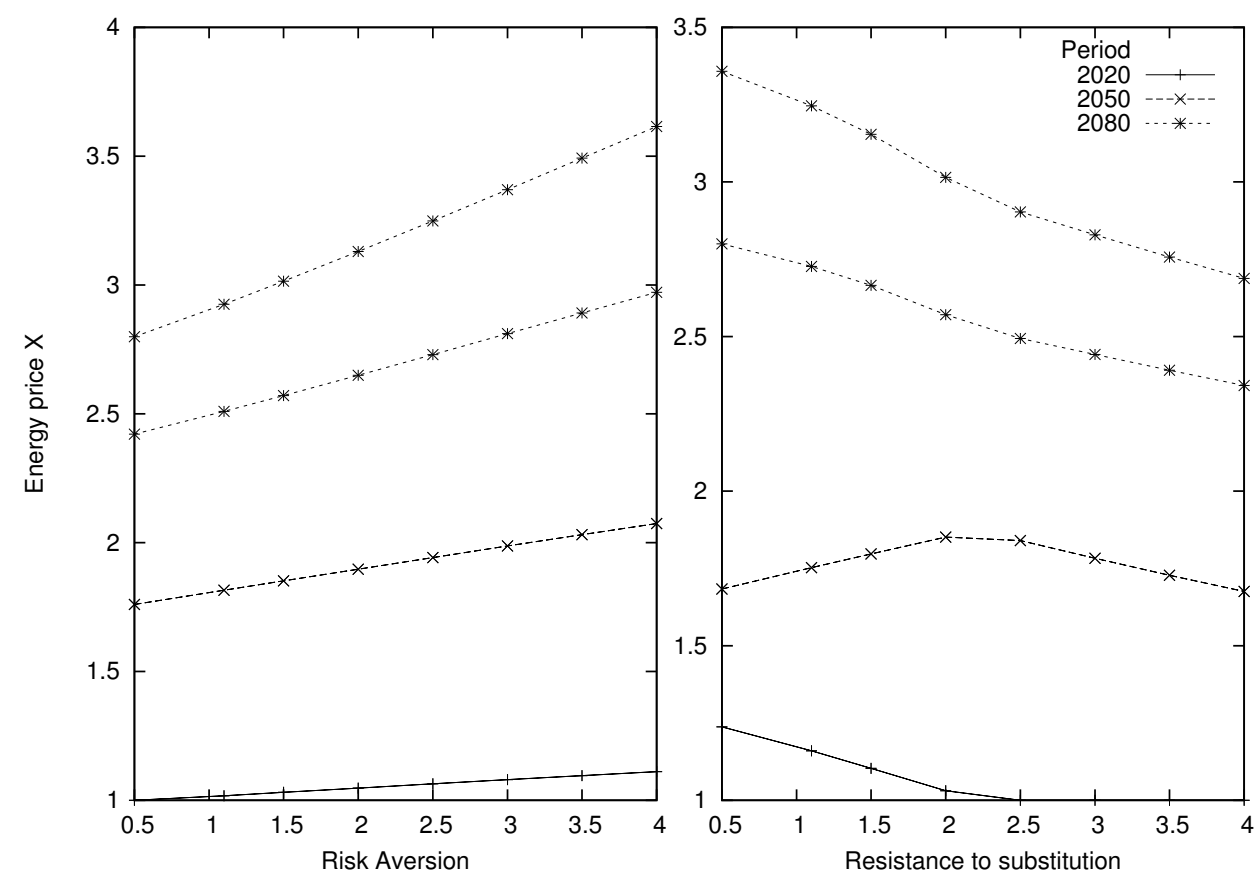

Figure 1: Optimal energy price multiplier $X$ at period 2, 3 and 4 for varying preference functions parameters. The left panel varies risk aversion $\gamma$ keeping $\rho=1.5$ while the right panel varies $\rho$ keeping $\gamma=2.0$ 


\begin{tabular}{lllll}
\hline$\gamma$ (risk aversion) & 1.5 & 2.0 & 1.5 & 2.0 \\
$\rho$ (resistance to subst.) & 2.0 & 2.0 & 1.5 & 1.5 \\
\hline Discount 1\% & 3.1 & 4.8 & 10.3 & 12.0 \\
Discount $1.5 \%$ & 0.0 & 0.0 & 4.5 & 6.2 \\
\hline
\end{tabular}

Table 1: Joint sensitivity of $\left(X_{2}-1\right)$, i.e. the optimal tax in period 2, to the preferences parameters $\gamma, \rho$ and the discount rate.

Results show consistently that higher risk-aversion leads to an increase in the carbon tax. This illustrates our previous theoretical result where increased riskaversion leads to increase control provided that $\rho$ is larger than one. This is the case here since our baseline scenario uses $\rho=1.5$. Interestingly note that the effect of the risk-aversion is nearly linear. The effect of an absolute change in $\gamma$ thus does not depend on the initial value of risk-aversion. For instance, in 2050, for a 1 point variation in risk-aversion, e.g. a change of $\gamma$ from 1 to 2 , the optimal carbon tax would be increased by approximately $15 \%$.

Turning to the effect of resistance to substitution, the right panel shows that increasing $\rho$ generally leads to a decrease in optimal pollution control. There is an apparent exception in 2050 where optimal pollution control increases for low value of $\rho$. But this is more than compensated by a decrease in 2020 tax level, so it remains true that overall higher values for $\rho$ lead to a decreased optimal tax. Indeed the transfer of the tax load from 2020 to 2050 as $\rho$ increases is a logical result since the 2050 generation is richer, so higher $\rho$ means placing the burden on it instead of the 2020 period.

This shows that there are two effects to increasing $\rho$ : one is to decrease the overall level of pollution control, the other is to transfer the burden to the future. The order of magnitude of the effect of an absolute change in $\rho$ is globally the same as for a change in $\gamma$. For instance, in 2020, an increase in $\rho$ from 1 to 2 leads to a decrease in tax by about $15 \%$.

Sensitivity of results to joint variations in $\rho$ and $\gamma$ are reported in Table 1. Annual discount rates of 1 and 1.5 per cent are examined. Table 1 confirms that, all other things being equal, increasing risk aversion leads to higher control. Similarly, increasing resistance to intertemporal substitution or increasing the discount rate leads to lower control. Note though that a $1.5 \%$ discount rate together with a resistance to substitution parameter $\rho=2$ makes the positive tax constraint binding. As a result the optimal tax hits zero in that case and is not sensitive to risk aversion.

This table also illustrates the effect of risk-aversion within an EU framework, i.e. under the constraint $\gamma=\rho$. First observe that, with a $1 \%$ discount rate, increasing risk aversion alone from 1.5 to 2 leads to a slightly increased carbon tax from $10.3 \%$ to $12.0 \%$. Yet, under the constraint $\gamma=\rho$, increasing risk aversion from $\gamma=1.5(=\rho)$ to $\gamma=2.0(=\rho)$ would lead to a decrease, and not an increase, in the carbon tax from $10.3 \%$ to $4.8 \%$.

As a result, the EU model predicts a negative impact of risk-aversion on pollution control. This is paradoxical, but the explanation is obviously that it 
moves the two parameters simultaneously and the negative effect of $\rho$ more than offsets the positive effect of risk-aversion $\gamma$. Hence, the EU model may lead to understand wrongly the effect of the so-called risk-aversion parameter on the climate policy. More generally, since the effect of one parameter is counterbalanced by the effect of the other parameter, the EU model may misinterpret lack of sensitivity of the parameters $\gamma$ and $\rho$. Here the sensitivity of optimal tax to a cross variation in the parameters is only 40 to 60 percent larger than the sensitivity to a joint variation.

To summarize, these numerical results do correspond to the theoretical results. First, increasing risk aversion $\gamma$ has a positive effect on the control rate. Second, increasing resistance to intertemporal substitution $\rho$ decreases the control rate. Third, and consequently, the classical EU model which accounts for the simultaneous variation of these two parameters, may lead to an misunderestimate of the effect of one parameter alone. This may explain, in turn, why previous climate change models, such as DICE, found little sensitivity of climate policy to risk-aversion.

\section{Conclusion}

In this paper, we explained how to use the generalized Kreps-Porteus recursive preferences in an integrated assessment model of global climate change. This kind of social objective function cuts the link between risk aversion $\gamma$ and resistance to intertemporal substitution $\rho$. This is in contrast with most previously published climate policy models, that maximize the discounted sum of logarithmic expected utility, corresponding to the $\rho=\gamma=1$ case.

Theoretical and empirical results about the sensitivity of the optimal energy consumption path show that these two parameters have opposite effects. Larger risk aversion increases optimal pollution control: provided that $\rho$ is not too low, more risk aversion $\gamma$ leads to less emissions in the short term. Larger resistance to substitution $\rho$ induces less pollution control. This is an income effect stating that assuming expected economic growth, higher $\rho$ leads to more emissions in the short term. This is equivalent to increasing the discount rate, which also implies a higher level of optimal pollution in the near-term.

Consequently, constraining $\rho=\gamma$ leads to underestimating the sensitivity of optimal results to the shape of the social welfare function. Recursive preferences thus contribute to the discussion on discounting in large risks cost-benefit analysis. It provides an analytical framework for precautionary-principle motivated preferences: low resistance to substitution with high risk aversion leads to the largest carbon tax levels.

\section{Acknowledgments}

We acknowledge CNRS and CEA support for this paper. We thank anonymous referees for very useful comments together with Anne Épaulard, Christian Gol- 
lier, Jean-Charles Hourcade, Alan Manne, Aude Pommeret and seminar participants in years 1999 and 2000 at GREEN (Québec), AFSE (Toulouse), CIRED (Paris), EAERE (Oslo) and Ateliers Columbia-CIRANO (Montréal). Remaining errors are only ours. 


\section{Annex 1 Theoretical resolution}

The objective is to find

$$
\max _{m_{1}, m_{2}} U_{1}=\left(\left(y_{1}+m_{1}-r m_{0}\right)^{1-\rho}+\beta\left(\mathrm{E}\left(y_{2}-r m_{1}+m_{2} \widetilde{x}\right)^{1-\gamma}\right)^{\frac{1-\rho}{1-\gamma}}\right)^{\frac{1}{1-\rho}}
$$

The dynamic optimization program (10) is solved in two steps backward. The first step is to find $m_{2}^{*}$ maximizing $\left(\mathrm{E}\left(y_{2}-r m_{1}+m_{2} \widetilde{x}\right)^{1-\gamma}\right)^{\frac{1}{1-\gamma}}$. The firstorder condition for $m_{2}^{*}$ gives:

$$
m_{2}^{*}=\left(y_{2}-r m_{1}\right) k, \text { with } k \text { defined by } \mathrm{E} \widetilde{x}(1+k \widetilde{x})^{-\gamma}=0
$$

This implies that the certainty equivalent of future consumption is:

$$
\mu\left(\widetilde{c}_{2}^{*}\right)=\left(y_{2}-r m_{1}\right) \mu[\gamma], \text { with } \mu[\gamma]=\left(\mathrm{E}(1+k \widetilde{x})^{1-\gamma}\right)^{1 /(1-\gamma)}
$$

Note that $\mu[\gamma]$ is positive and does not depends on $m_{1}$. This can be seen as the certainty equivalent of future risk by unit of consumption

The second step is to solve the period 1 problem, that is maximize $U_{1}$ given (12). The first order condition for $m_{1}^{*}$ gives:

$$
\left(y_{1}+m_{1}^{*}-r m_{0}\right)^{-\rho}-\beta r(\mu[\gamma])^{1-\rho}\left(y_{2}-r m_{1}^{*}\right)^{-\rho}=0
$$

that is

$$
m_{1}^{*}=\frac{r m_{0}-y_{1}+y_{2} \mu[\gamma](\beta r \mu[\gamma])^{-1 / \rho}}{1+r \mu[\gamma](\beta r \mu[\gamma])^{-1 / \rho}}
$$

One can easily check that second order conditions are satisfied, so that $m_{1}^{*}$ is the level of first-period stock that maximizes social welfare. Therefore, using $c_{1}^{*}=y_{1}+m_{1}^{*}-r m_{0}$, it is direct to see that the optimal consumption level in first period is the one given in (4).

From (4), it is then straightforward to obtain:

$$
\begin{aligned}
\frac{\partial \log \left(c_{1}^{*}\right)}{\partial \gamma} & =\frac{\rho-1}{\mu[\gamma] \rho\left(1+\beta^{-1 / \rho}(\mu[\gamma] r)^{1-1 / \rho}\right)} \times \mu^{\prime}[\gamma] \\
\frac{\partial \log \left(c_{1}^{*}\right)}{\partial \rho} & =\frac{\log (\beta r \mu[\gamma])}{\rho^{2}\left(1+\beta^{-1 / \rho}(\mu[\gamma] r)^{1-1 / \rho}\right)}
\end{aligned}
$$

Focus first on the effect of relative risk aversion. Using the envelope theorem, it is easy to find that the certainty equivalent $\mu[\gamma]$ decreases with risk-aversion $\gamma$, that is $\mu^{\prime}[\gamma]<0$. With equation (15), this implies that higher risk aversion increases emissions in period if and only if resistance to substitution is sufficiently small, $\rho<1$.

Turn now to the effect of resistance to substitution. Equation (16) shows that $\partial \log \left(c_{1}^{*}\right) / \partial \rho$ has the sign of $\log (\beta r \mu[\gamma])$. Therefore, first-period emissions increase with resistance to substitution if and only if $\beta r \mu[\gamma]>1$. 


\section{Annex 2 DIAM-ULTRAL model}

This model can be used freely and the source downloaded at the author's homepage.

\section{Variables}

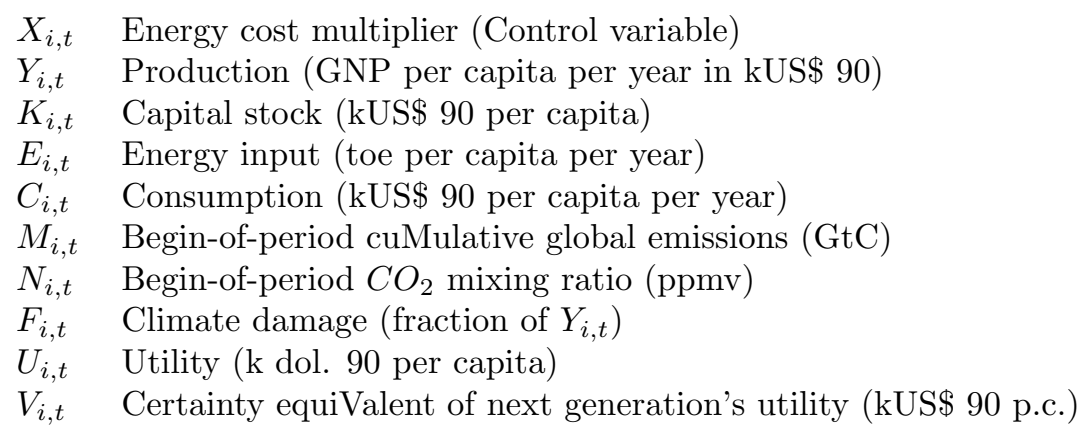

\section{Equations}

DIAM-ULTRAL maximizes $U_{H, 1}$ under constraints $17-25$, the stochastic dynamic programming constraint $X_{L, t}=X_{H, t}$ for all $t \leq 3$ and the final stationarity condition $N_{i, 4}=N_{i, 3}$.

$$
\begin{aligned}
Y_{i, t} / Y^{1} & =a_{t} F_{i, t}\left(K_{i, t} / K_{1}\right)^{k}\left(E_{i, t} / E^{1}\right)^{e} \\
C_{i, t} & =\left(1-s_{t}\right) Y_{i, t} \\
Y_{i, t} / Y^{1} & =p_{t} X_{i, t} E_{i, t} / E^{1} \\
K_{i, t+1} l_{t+1} & =\left(\mu K_{i, t}+\nu s_{t} Y_{i, t}\right) l_{t} \\
M_{i, t+1} & =M_{i, t}+\delta \chi_{t} E_{i, t} l_{t} \\
N_{i, t} & =\alpha_{t}^{0}+\alpha_{t}^{1} M_{i, t} \\
F_{i, t} & =1-\Delta_{i} \frac{N_{i, t}-275}{275} \\
U_{i, t}^{1-\rho} & =(1-\beta)\left(l_{t} / l_{4}\right)^{\rho} C_{i, t}^{1-\rho}+\beta V_{i, t}^{1-\rho} \\
V_{i, t} l_{t} & = \begin{cases}U_{i, t+1} l_{t+1} \\
{\left[\pi\left(l_{t+1} U_{H, t+1}\right)^{1-\gamma}+(1-\pi)\left(l_{t+1} U_{L, t+1}\right)^{1-\gamma}\right]^{\frac{1}{1-\gamma}}} & t=3(25) \\
C_{i, 4} l_{4}\left(\mu K_{i, 4}+\nu s_{4} Y_{i, 4}\right) / K_{i, 4} & t=4\end{cases}
\end{aligned}
$$




\section{Baseline parameters (Corresponds to IPCC A2 scenario)}

$\begin{array}{lll}k & \text { Elasticity of capital } & 0.36 \\ e & \text { Elasticity of energy } & 0.04 \\ l_{t} & \text { World population } & 5.26,8.19,11.30,12.35 \text { billions } \\ a_{t} & \text { Technical progress coefficient } & 1.00,1.22,1.55,1.62 \\ s_{t} & \text { Saving ratio } & 0.30,0.30,0.25,0.25 \\ p_{t} & \text { Baseline increase in the energy input cost } & 1.00,1.07,1.33,1.79 \\ Y^{1} & \text { Production per capita in 1990 without damages } & 3.82 \mathrm{k} \$ 90 \\ E^{1} & \text { Energy per capita in 1990 without reduction } & 1.21 \mathrm{toe} \\ K_{1} & \text { Initial capital } & 11.46 \mathrm{k} \$ 90 \\ \mu & \text { Inter-period depreciation of capital } & 0.215 \\ \nu & \text { Inter-period accumulation of investment } & 16.14 \\ \quad & & \\ \beta & \text { Inter-period utility discount factor } & 0.74 \\ \rho & \text { Resistance to intertemporal substitution } & 1.5 \\ \gamma & \text { Relative risk-aversion } & 2.0 \\ & \quad \text { Duration of a period } & 30 \mathrm{years} \\ \delta & \text { Carbon content of energy } & 0.97,0.91,0.84,1.17 \mathrm{tC} / \mathrm{toe} \\ \chi_{t} & \text { Intercept of the carbon-cycle relation } & 354,371,390,297 \mathrm{ppmv} \\ \alpha_{t}^{0} & \text { Slope of the carbon-cycle relation } & 0.154,0.129,0.278,0.168 \mathrm{ppmv} / \mathrm{toe} \\ \pi & \text { Probability for a negative impact } & 0.25 \\ \Delta & \text { Climate change productivity loss at } 550 \mathrm{ppmv} & 0.08 \mathrm{if} \mathrm{L}, 0.30 \mathrm{if} \mathrm{H}\end{array}$




\section{References}

[1] Kenneth J. Arrow. Discounting, Morality and Gaming, chapter 2. Resources for the Future, Washington, DC, 1999.

[2] R. B. Barsky, F. T. Juster, M. Kimball, and M. D. Shapiro. Preferences parameters and behavioral heterogeneity: An experimental approach in the health and retirement study. Quarterly Journal of Economics, pages 537-579, 1997.

[3] L. G. Epstein and S. E. Zin. Substitution, risk-aversion and the temporal behavior of consumption and asset returns: An empirical analysis. Journal of Political Economy, 99:263-286, 1991.

[4] Christian Gollier. Discounting an uncertain future. Journal of Public Economics, 85:149-166, 2002.

[5] Minh Ha-Duong, Michael J. Grubb, and Jean-Charles Hourcade. Influence of socioeconomic inertia and uncertainty on optimal $\mathrm{CO}_{2}$-emission abatement. Nature, 390:270-274, 1997.

[6] Minh Ha-Duong and Nicolas Treich. Recursive intergenerational utility in global climate risk modeling. NOTA DI LAVORO 92.99, Fondazione Eni Enrico Mattei, 2000. Also presented at EMF/IEW meeting in Stanford, July 2000, at Journees de l'IDEI, Toulouse, 16-17 juin 1999. Also presented at the EUREQUA, Paris environmental Economics seminar, 2 dec 1999.

[7] R. E. Hall. Intertemporal substitution in consumption. Journal of Political Economy, 96:338-357, 1988.

[8] Working Group III IPCC, editor. Summary for Policymakers of IPCC special report on emissions scenarios. UNEP - WMO, 2000.

[9] D. Kreps and E. Porteus. Temporal resolution of uncertainty and dynamic choice theory. Econometrica, 46:185-200, 1978.

[10] William D. Nordhaus. Managing the Global Commons. MIT Press, 1994.

[11] M. Normandin and P. Saint-Amour. Substitution, risk aversion, taste shocks and equity premia. Journal of Applied Econometrics, pages 265-281, 1998.

[12] M. Ogaki and M. R. Reinhart. Measuring intertemporal substitution: The role of durable goods. Journal of Political Economy, pages 1078-1098, 1998.

[13] L. Selden. A new representation of preferences over 'certain $\times$ uncertain' pairs: The 'ordinal certainty equivalent hypothesis'. Econometrica, 16:1045,1060, 1978.

[14] M. Weitzman. Why the far-distant future should be discounted at its lowest possible rate? Journal of Environmental Economics and Management, 36:210-208, 1998. 